\title{
Abnormal Division of Radial Nerve Encountered during Fixation of Fracture of Shaft of Humerus through Anterior Approach
}

\author{
Periakaruppan Ramasamy¹, Masilamani Kalaivanan²
}

\begin{abstract}
Variation in the branching pattern of the posterior cord of the brachial plexus is a rare occurrence when compared with the variations in the branching pattern of the medial and lateral cords. Even more rarer is the variation in the branching pattern of the radial nerve. This article is a report of the observation made during surgical fixation of humerus shaft fracture through the anterior approach wherein radial nerve was seen as anterior and posterior divisions rather than a single nerve bundle. The anterior division continued as the main radial nerve and posterior division gave branches to the triceps muscle. An orthopedic surgeon should be aware of radial nerve divisions while exposing the humerus shaft and butterfly fragment for fracture fixations. Bone clamps should be applied with caution.

Keywords: Early rehabilitation, Fracture, Functional outcome.

TNOA Journal of Orthopedics and Joint Surgery (2021): 10.5005/jp-journals-10079-1034
\end{abstract}

\section{INTRODUCTION}

The brachial plexus is a network of nerves formed by the ventral rami from $\mathrm{C} 5-8$ and $\mathrm{T} 1$. The radial nerve is the continuation of the posterior cord in the axilla with a root value of $C 5,6,7,8$, and T1. Anomalies may occur with the "formation" or "branching" of the plexus. ${ }^{1,2}$ Anomaly in the "formation" starts from the level of formation (pre or post fixed ${ }^{3}$ ) and extends to trunks, divisions, and cords. An anomaly in the "branching" pattern of the cords is the more common than the "formation" anomaly. Arrangement of the terminal branches shows very little variation. ${ }^{1}$ In this report, a rare variation in the branching pattern of radial nerve (terminal branch) is reported.

\section{Case Description}

A 65-year-old man presented with a "right" middle third humerus shaft fracture with intact radial nerve function. He underwent plate fixation for this fracture via anterior approach. Surgery was carried out under general anesthesia. Anterior midline incision was made over right arm. Biceps was reflected and brachialis was split. While exposing the proximal and distal ends of the fracture, radial nerve was seen close to the distal fragment but separated by a few muscle fibers from triceps (Fig. 1). The dissection was carried out to identify the butterfly fragment. Once this fragment was dissected out and brought into view, the radial nerve had come into the view. The radial nerve was seen as "two" divisions (Fig. 2). Further dissection was carried out proximally and distally to trace the divisions of the radial nerve within the limits of the skin incision that was planned for plating of humerus. The anterior division of the radial nerve was continuing as the regular radial nerve as it was crossing the lateral intermuscular septum and continued distally. The posterior division was seen giving branches to triceps muscle at regular intervals and was seen entering the triceps muscle distally. Nerve stimulator with sterile needle (Braun-used by an anesthesiologist) was used to stimulate the divisions of the radial nerve and to identify the muscle twitching. A current of $0.2 \mathrm{~mA}$ at $0.1 \mathrm{~ms}$ stimulation was used to elicit muscle response. Eliciting muscle response over anterior division

\footnotetext{
1,2Department of Orthopaedics, Kauvery Hospital, Trichy, Tamil Nadu, India
}

Corresponding Author: Periakaruppan Ramasamy, Department of Orthopaedics, Kauvery Hospital, Trichy, Tamil Nadu, India, Phone: +91 9842409298, e-mail: ramassamy@hotmail.com

How to cite this article: Ramasamy P, Kalaivanan M. Abnormal Division of Radial Nerve Encountered during Fixation of Fracture of Shaft of Humerus through Anterior Approach. TNOA J Orth Joint Surg 2021;3(2):80-81.

Source of support: Nil

Conflict of interest: None

resulted in contraction of extensor muscles of forearm and that of posterior division resulted in the contraction of triceps muscle only. Surgical fixation was carried out without causing any iatrogenic damage to the radial nerve (Fig. 3).

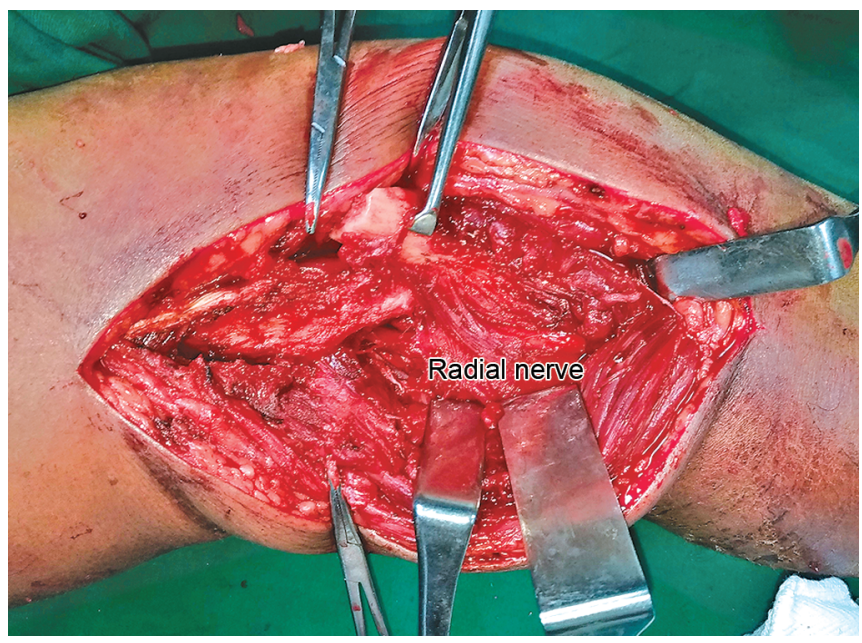

Fig. 1: Radial nerve seen during the course of dissection and identification of fractured bone fragments 


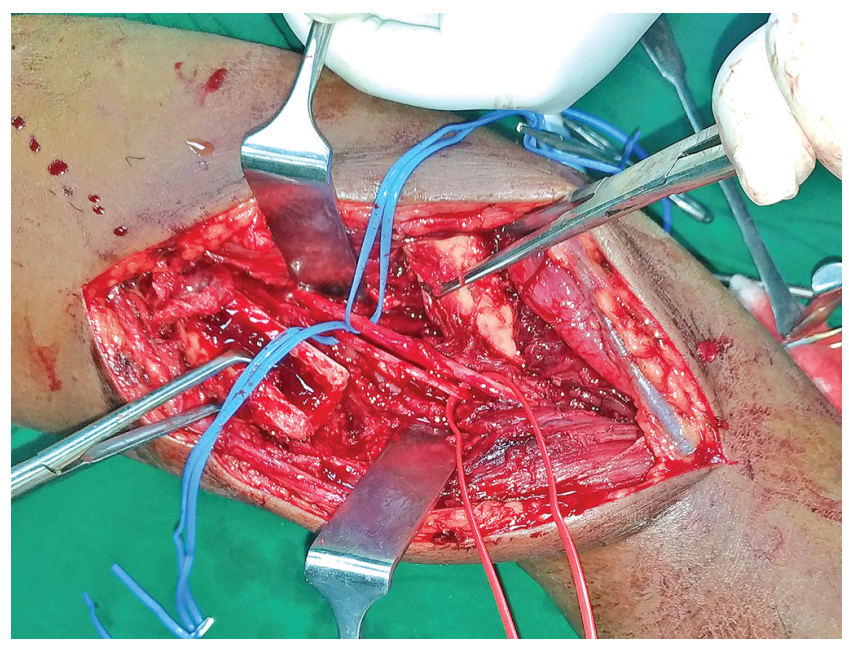

Fig. 2: Anterior and posterior divisions of radial nerve during dissection for butterfly fragment

\section{Discussion}

Among the "formation" of cords, variations have been noted often in the medial and lateral cords but less often in the posterior cord. ${ }^{1}$ Aktan et al. ${ }^{4}$ reported that posterior cord and radial nerve arose only from the union of posterior divisions of the inferior and middle trunks. Oluyemi ${ }^{5}$ reported that brachial plexus was noted with two cords (medial and lateral) and three abnormal communications. A branch from the posterior aspect of the medial cord divided into the radial and axillary nerves.

While considering the "branching pattern" of the cords, variations have been reported to a greater extent in lateral and medial cords rather than in the posterior cord. ${ }^{6}$ Ebenezer and Rathinam ${ }^{7}$ and Bertha et al. ${ }^{8}$ reported that the posterior cord was found to be divided into upper and lower divisions by an axillary arch and a common arterial trunk from the third part of the axillary artery. The upper-division of the posterior cord gave rise to the axillary nerve and the radial nerve. The lower division of the posterior cord gave rise to the upper and lower subscapular nerves and thoracodorsal nerve. Kuwar and Bilodi ${ }^{9}$ and Bhat ${ }^{10}$ reported that radial nerve arose as two roots from the posterior cord of brachial plexus. One of the roots was passing posterior to the subscapular artery and the other root anterior. Further down both the roots united to form the trunk of the radial nerve. Honma et al. ${ }^{11}$ reported that an aberrant axillary artery was found to penetrate the radial nerve from deep to superficial during its course. Muthoka et al. ${ }^{12}$ studied branching pattern of posterior cords in Kenyan population. He reported that only $10 \%$ showed classical branching pattern and formation of radial nerve from posterior cord or radial nerve itself did not show any variation.

Jamuna ${ }^{13}$ reported that the radial nerve originating from the posterior cord was found splitting into anterior and posterior divisions. Both the divisions had their course in the radial groove and the posterior division was giving off the branches which are expected to have origin from the main radial nerve. The anterior division continued as the main radial nerve in the radial groove and had a usual course in the rest of the upper limb.

In the current case report, radial nerve divisions were similar to the case report by Jamuna. ${ }^{13}$ The essential feature to be noted is that "all" the variations that have been described in the above said literature has been observed in "cadaveric dissections". The current case report is the "only" report of abnormal radial nerve division in

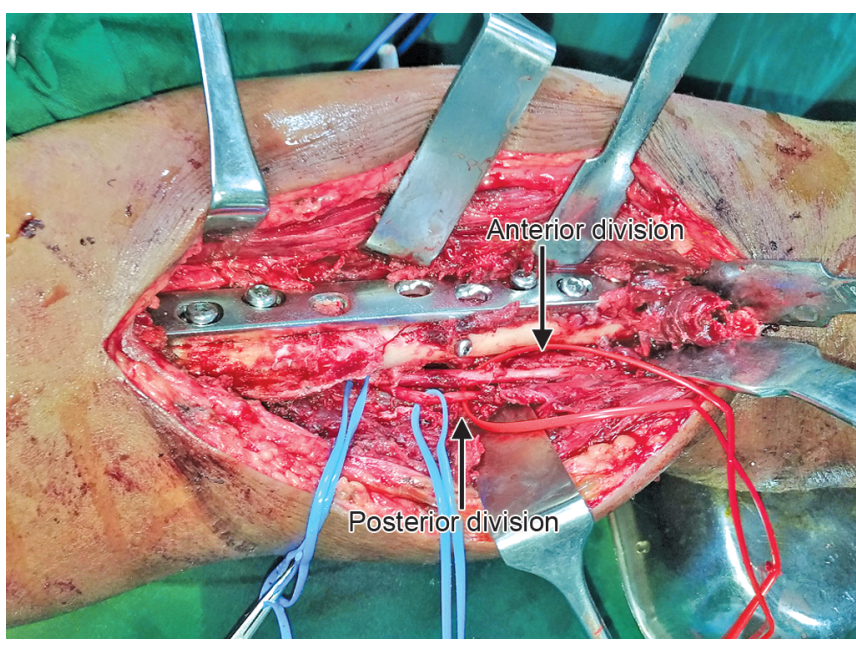

Fig. 3: Anterior and posterior divisions of radial nerve at the end of fracture fixation

"routine orthopedic surgical procedure". Orthopedic surgeon has to be aware of radial nerve divisions while exposing humerus shaft for fixations. Bone clamps should be applied with caution making sure that radial nerve, particularly when it has two divisions, does not get caught in-between the bone and the clamp.

\section{References}

1. Gupta M, Goyal N, Harjeet. Anomalous communications in the branches of brachial plexus. J Anat Soc India 2005;54:22-25.

2. Gopal K, Anurag, Singh O. Anatomical variations of brachial plexus: anomalous branching pattern. International Journal of Research in Medical Sciences. Int J Res Med Sci 2016;4(8):3376-3380. DOI: 10.18203/2320-6012.jjrms20162297.

3. Emamhadi M, Chabok SY, Samini F. Anatomical variations of brachial plexus in adult cadavers; a descriptive study. Arch Bone Jt Surg 2016;4(3):253-258.

4. Aktan ZA, Ozturk L, Bilge $O$, et al. A cadaveric study of the anatomic variations of the brachial plexus nerves in the axillar region and arm. Turk J Med Sci 2001;31:147-150.

5. Oluyemi KA, Adesanya OA, Ofusori DA, et al. Abnormal pattern of brachial plexus formation: an original case report. Internet J Neurosurg 2007;4(2):1-5.

6. Chaudhary P, Singla R, Arora K, et al. Formation and branching pattern of cords of brachial plexus- a cadaveric study in north Indian population. Int J Anat Res 2014;2(1):225-233. ISSN 2321-4287.

7. Ebenezer DA, Rathinam BAD. Rare multiple variations in brachial plexus and related structures in the left upper limb of a Dravidian male cadaver. Anat Cell Biol 2013;46(2):163-166. DOI: 10.5115/ acb.2013.46.2.163.

8. Bertha A, Kulkarni NV, Maria A, et al. Entrapment of deep axillary arch by two roots of radial nerve - an anatomical variation. J Anat Soc India 2009;58:40-43.

9. Kuwar RB, Bilodi AK. Clasping of subscapular artery by radial nerve. Kathmandu Univ Med J 2007;5:253-255.

10. Bhat KMR, Girijavallabhan V. Variation in the branching pattern of posterior cord of brachial plexus. Neuroanatomy 2008;7:10-11.

11. Honma S, Kawai K, Koizumi M, et al. An aberrant axillary artery penetrating the origin of the radial nerve from deep to superficial. Ann Anat 2004;186(2):153-156. DOI: 10.1016/S0940-9602(04) 80031-1.

12. Muthoka JM, Sinkeet SR, Shahbal SH, et al. Variations in branching of the posterior cord of brachial plexus in a Kenyan population. J Brach Plexus Periphe Ner Inj 2011;6(01):1. DOI: 10.1186/1749-7221-6-1.

13. Jamuna M. A rare variation of the branching pattern of radial nerve. Int J Anat Var 2011;4:22-24. 